\title{
COVID-19 in a Patient Newly Diagnosed with AIDS: Asymptomatic Disease
}

\author{
Manuel Serrano Martins ${ }^{1}$, Fabiana Pimentel ${ }^{1}$, Wildemar Costa $^{1}$, Claudia Fernandes $^{2}$ \\ ${ }^{1}$ Department of Internal Medicine, Torres Vedras Unit, West Hospital Center, Torres Vedras, Portugal \\ ${ }^{2}$ Department of Infectious Diseases, Torres Vedras Unit, West Hospital Center, Torres Vedras, Portugal
}

Doi: 10.12890/2021_002204 - European Journal of Case Reports in Internal Medicine - ๑ EFIM 2021

Received: 09/12/2020

Accepted: $11 / 12 / 2020$

Published: 22/01/2021

How to cite this article: Serrano Martins M, Pimentel F, Costa W, Fernandes C. COVID-19 in a patient newly diagnosed with AIDS: asymptomatic disease. EJCRIM 2021;8: doi:10.12890/2021_002204.

Conflicts of Interests: The Authors declare that there are no competing interests.

This article is licensed under a Commons Attribution Non-Commercial 4.0 License

\section{ABSTRACT}

The COVID-19 pandemic has posed new challenges for the scientific community. The behaviour of the virus and outcomes in people living with HIV have not yet been properly studied. We report the case of a 34-year-old man with newly diagnosed HIV infection stage 4 and asymptomatic SARS-CoV-2 infection. Although immunocompromised patients are classified as being at high risk of developing severe COVID-19, HIV-related immunosuppression may have a protective role.

\section{LEARNING POINTS}

- It is important to remember that not every respiratory infection during this pandemic is due to SARS-CoV-2.

- We need more studies to elucidate the behaviour of COVID-19 in people living with HIV.

- There is still doubt about the best time for antiretroviral therapy (ART) initiation in this context.

\section{KEYWORDS}

COVID-19, SARS-CoV-2, HIV, AIDS, immunosuppression

\section{INTRODUCTION}

Severe acute respiratory syndrome coronavirus 2 (SARS-CoV-2) has caused a global pandemic with over 65 million cases reported and more than 1.5 million deaths so far ${ }^{[1]}$. Despite scientific progress and a rapidly accumulating literature, many questions about the disease are still unanswered, for instance it is not known if people living with HIV (PLWH) are at higher risk of developing severe COVID-19. There is no scientific evidence that immunosuppressed patients are at higher risk of developing severe disease; indeed, it has been proposed that in $\mathrm{PLWH}$, antiretroviral therapy (ART) and persistent immune dysregulation could confer some protection ${ }^{[2]}$.

\section{CASE DESCRIPTION}

We report the case of a 34-year-old man, with no relevant medical history, who presented to the emergency room with a 2-week progressive fever associated with cough, bloody sputum, anterior chest pain and dyspnoea. The patient also complained of asthenia, anorexia, dysphagia and a weight loss of about $10 \mathrm{~kg}$ over the previous 3 months. Upon physical examination, he was febrile (temperature $39.6^{\circ} \mathrm{C}$ ), hypoxaemic $\left(\mathrm{SpO}_{2} 87 \%, \mathrm{FiO}_{2} 21 \%\right)$ and tachycardic (140 bpm). Peripheral blood samples showed haemoglobin $12.6 \mathrm{mg} / \mathrm{dl}$, white blood cell count of 6980 cells $/ \mathrm{mm} 3$ (neutrophils 4970 cells/mm3, lymphocytes 1340 cells $/ \mathrm{mm}^{3}$ ) and C-reactive protein 12 mg/dl. Chest radiography showed bilateral reticular interstitial opacities, while chest computed tomography revealed a bilateral diffuse ground-glass pattern and basal bilateral consolidations. Sputum culture grew methicillin-susceptible Staphylococcus aureus (MSSA). Serologies were positive for HIV-1 with a CD4+ count of 7 cells/mm3 and viral load of 111,000 copies/ml. Bronchoscopy was performed and bronchoalveolar lavage fluid culture was positive for Pneumocystis jiroveci. Two nasopharyngeal swabs were taken and excluded SARS-CoV-2 infection. 
The patient was admitted with the diagnosis of HIV infection stage C3/AIDS, bilateral pneumonia due to P. jiroveci and MSSA superinfection and wasting syndrome. During hospitalization, because of respiratory failure he was transferred to an ICU and received mechanical ventilation, being extubated on the 3rd day. He underwent treatment with cotrimoxazole, levofloxacin and prednisolone for 4 weeks with full respiratory recovery. Because of contact with a COVID-19-positive case during hospitalization, a third nasopharyngeal swab was taken but was negative for SARS-CoV-2. The patient was discharged with the indication to repeat the swab on the 7th day after the referred contact. Four days after discharge, the patient was readmitted with a positive COVID-19 swab. He had no COVID-19 symptoms. Laboratory studies showed leukopenia 2630 cells $/ \mathrm{mm}^{3}$ (neutrophils 1320 cells $/ \mathrm{mm}^{3}$, lymphocytes $610 \mathrm{cells} / \mathrm{mm}^{3}$ ), haemoglobin $12.5 \mathrm{mg} / \mathrm{dl}$, thrombocytopenia of 127,000 platelets $/ \mathrm{mm} \underline{3}$, D-dimers $204 \mathrm{ng} / \mathrm{ml}$, and C-reactive protein $0.5 \mathrm{mg} / \mathrm{dl}$. It was decided to defer ART initiation. After 2 weeks of hospitalization without symptoms and no specific COVID-19 treatment, although the patient was still positive for SARS-CoV-2, ART was initiated with bictegravir $50 \mathrm{mg} /$ emtricitabine $200 \mathrm{mg} /$ tenofovir alafenamide $25 \mathrm{mg}$ and he was discharged with a recommendation for infectious diseases consultation. The patient remained SARS-CoV-2 positive for 2 months but was always asymptomatic.

\section{DISCUSSION}

Despite being seriously immunocompromised (only 7 CD4+ cells $/ \mathrm{mm}^{3}$ ), this patient did not develop SARS-CoV-2-related illness.

Although immunocompromised patients are classified as a high-risk group for developing severe COVID-19, this case, along with other case reports, raises the question of whether HIV-related immunosuppression could play a protective role. In fact, the persistent immune dysregulation present in HIV patients might play a part, preventing the cytokine storm associated with severe COVID-19. However, according to the few studies in the literature, the incidence and outcome of COVID-19 in PLWH is similar to that in the general population ${ }^{[2,3]}$. The timing of when to start ART in this patient was a challenge, since there are no guidelines providing information on the safety of initiating this therapy in a patient positive for SARS-CoV-2. ART was not started during the first 2 weeks of COVID-19 in case systemic inflammatory response syndrome (SIRS) developed. Although the patient remained positive for SARS-CoV-2, on discharge he was still asymptomatic and severely immunosuppressed, so it was decided to initiate ART under close surveillance, with no infectious exacerbation observed.

The patient remained SARS-CoV-2 positive for 2 months, a much longer period than normal (average 20 days), a pattern noticed in other immunocompromised patients. This could be possibly explained by a diminished viral clearance rate in these particular patients ${ }^{[4]}$. More time and studies are needed to address all the questions regarding COVID-19 in immunosuppressed patients.

\section{REFERENCES}

1. World Health Organization. WHO coronavirus disease (COVID-19) dashboard. Geneva: World Health Organization; 2020. Available at https://covid19.who.int/ (accessed 13 Jan 2021).

2. Inciarte A, Gonzalez-Cordon A, Rojas J, Torres B, de Lazzari E, de la Mora L, et al. Clinical characteristics, risk factors, and incidence of symptomatic coronavirus disease 2019 in a large cohort of adults living with HIV: a single-center, prospective observational study. AIDS 2020;34(12):1775-1780.

3. Fung M, Babik JM. COVID-19 in immunocompromised hosts: what we know so far. Clin Infect Dis 2020 Jun 27; ciaa863. doi: 10.1093/cid/ciaa863. Online ahead of print.

4. Xiao T, Wang Y, Yuan J, Ye H, Wei L, Liao X, et al. Early viral clearance and antibody kinetics of COVID-19 among asymptomatic carriers. medRxiv May 02, 2020; doi: https://doi. $\operatorname{org} / 10.1101 / 2020.04 .28 .20083139$. 